\title{
Für Diabetiker ist Sport anstrengender
}

\author{
Ältere Typ-2-Diabetikerinnen müssen mehr Aufwand betreiben, um den gleichen sportlichen Effekt \\ zu erreichen wie Kontrollpersonen. Das sollten Trainingspläne gerade zu Beginn berücksichtigen.
}

Ist ein inaktiver Lebensstil Ursache für Diabetes, oder könnte er auch die Folge einer Insulinresistenz und Hyperglykämie sein? Diese Frage wurde in einer Studie mit postmenopausalen Frauen zwischen 50 und 75 Jahren untersucht. 26 von ihnen hatten Typ-2-Diabetes, 28 dienten als Kontrollen. Mit dem Borg Rating of Perceived Exertion wurde der Aufwand für submaximale Leistungen auf einem Fahrradergometer gemessen. Angestrebt wurden 30 Watt Leistung oder $35 \%$ des individuell gemessenen maximalen Sauerstoffverbrauchs $\left(\mathrm{VO}_{2} \max \right)$.

Daneben wurden metabolische Parameter wie die Laktatkonzentration, aber auch Ernährungsverhalten, Taillenum- fang und Body-Mass-Index und das Verhältnis von Muskel- zu Fettmasse mit Hilfe des DXA-Scans erfasst. Metabolische Faktoren wie Insulin und Glukose wurden analysiert, um die Insulinresistenz mittels HOMA zu bestimmen.

Bei Diabetikerinnen war der $\mathrm{VO}_{2} \max$ niedriger als bei Gesunden. Der Aufwand für die körperliche Aktivität war bei Diabetes nicht signifikant größer, aber die gemessenen Laktatspiegel waren deutlich höher. Der größere Aufwand für körperliche Aktivität war mit höherem Laktat, höherer Herzfrequenz und Bluthochdruck assoziiert.

- Huebschmann AG et al. Type 2 diabetes exaggerates exercise effort and impairs exercise performance in older women BMJ Open Diabetes Res Care. 2015;3:e000124

\section{KOMMENTAR}

In der Zusammenschau sind Diabetiker durch ihre Physiologie benachteiligt, körperlich aktiv zu sein. Das äußert sich in einem höheren Aufwand (Laktase, Herzfrequenz, Blutdruck) beim Erreichen des gleichen Levels an körperlicher Aktivität. Das wiederum ist eine signifikante Einstiegshürde für Sport. Die Autoren beschreiben in ihrer Diskussion sehr deutlich, dass diese Barriere abtrainierbar ist - durch das langsame Heranführen an körperliche Aktivität.

Ein Erfolgsmodell könnte ggf. sein, damit zu beginnen, 5.000, 6.000 und letztendlich auch 10.000 Schritte am Tag zu laufen und das zu nutzen, um langsam einer sportlichen Betätigung entgegen zu laufen.

Prof. Dr. med. P. Schwarz

\section{Nach zwei Tagen enthüllt sich die Quelle der Schmerzen}

Ein 75-jähriger Mann mit Hyperlipidämie und Hypertonie kam wegen neuaufgetretener, stechender Schmerzen in der linken Thoraxhälfte in die Nothilfe. Die Schmerzen strahlten in den linken Arm aus, waren nicht belastungsabhängig und konnten nicht durch eine Palpation der Brustwand ausgelöst oder verstärkt werden. Das EKG war unauffällig, bei dem Patienten be-
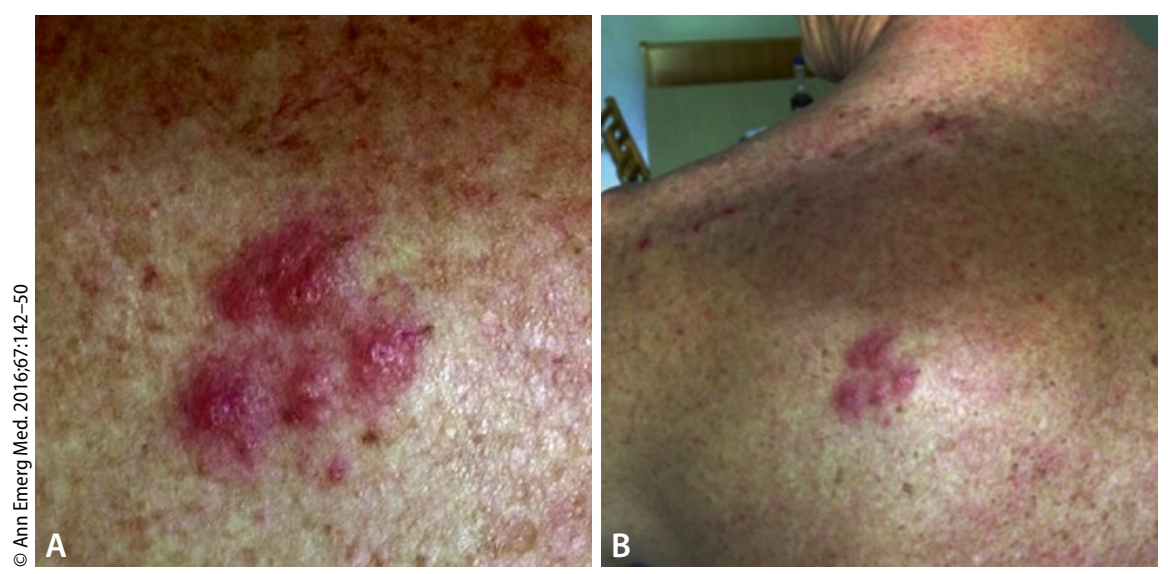

Vesikuläres, druck- und berührungsempfindliches Exanthem am Rücken. stand keine bekannte koronare Herzkrankheit. Auch die initialen und nach sechs Stunden kontrollierten Troponin-Konzentrationen waren normal. Röntgenthorax und Abdomensonografie fielen ebenso wie das Labor unauffällig aus. Der Patient wurde zunächst nach Hause geschickt. Am nächsten Vormittag hatte sich der Schmerzcharakter nicht wesentlich geändert.

Ein Belastungs-EKG fiel ebenfalls unauffällig aus. Des Rätsels Lösung offenbarte sich zwei Tage nach der ersten Vorstellung, als am Rücken links ein vesikuläres, druck- und berührungsempfindliches Exanthem erschienen war (Abb. A, B). Nun war klar, dass es sich um ein Schmerzphänomen bei einem Zoster im Bereich der Thoraxwand gehandelt hatte, das dem typischen Exanthem um zwei Tage vorausgegangen war.

Etwa zwei Drittel der Zoster-Patienten spüren 2-5 Tage vor Auftreten des Exanthems Schmerzen von meist brennendem Charakter. Treten diese im Thoraxbereich auf und besteht wegen des Alters und kardiovaskulärer Risikofaktoren der Verdacht auf eine koronare Herzkrankheit, so verursachen diese Patienten erhebliche diagnostische Probleme. In seltenen Fällen kann der Zoster ausschließlich Schmerzen und kein Exanthem hervorrufen (Zoster sine herpete).

Prof. Dr. med. H. S. FüeßI

- Lintner S, Welke S, Wiedermann CJ. Elderly Man With Chest Pain. Ann Emerg Med. 2016;67:142-50 